\title{
ARTICLE
}

\section{The effect of type II toxin-antitoxin systems on methicillin- resistant Staphylococcus aureus persister cell formation and antibiotic tolerance}

\author{
Mandana Hosseini' ${ }^{1}$, Jamileh Nowroozi', Nour Amirmozafari²* \\ 'Department of Microbiology, Tehran North Branch, Islamic Azad University, Tehran, Iran \\ ${ }^{2}$ Department of Microbiology, School of Medicine, Iran University of Medical Sciences, Tehran, Iran
}

\begin{abstract}
Persister cells are defined as a subpopulation of bacteria in a dormant state with the ability to reduce bacterial metabolism and they are involved in antibiotic tolerance. Toxin-antitoxin (TA) systems have been previously suggested as important players in persistence. Therefore, this study aimed to study the involvement of TA systems in persister cell formation in methicillin-resistant Staphylococcus aureus following antibiotic exposure. Using TADB and RASTA database, two type II TA systems including MazF/MazE and RelE/RelB were predicted in S. aureus. The presence of these TA genes was determined in 5 methicillin-resistant $S$. aureus isolates and the standard strain S. aureus subsp. aureus N315 using PCR method. To induce persistence, isolates were exposed to lethal doses of ciprofloxacin and the expression of the studied TA system genes was measured after $5 \mathrm{~h}$ using Real-Time PCR. According to our results, all the studied isolates harbored the TA system genes. S. aureus was highly capable of persister cell formation following exposure to sub-MIC of ciprofloxacin and RT-qPCR showed a significant increase in the expression of the MazEF and RelBE loci, indicating their potential role in antibiotic tolerance. Considering the importance of antibiotic tolerance, further studies on persister cell formation and TA systems involved in this phenomenon are required to efficiently target these systems.

Acta Biol Szeged 65(1):113-117 (2021)
\end{abstract}

\section{KEY WORDS}

methicillin resistance persister cells real-time PCR Staphylococcus aureus TA systems

\section{ARTICLE INFORMATION}

Submitted

13 June 2021.

Accepted

1 July 2021

*Corresponding author

E-mail: amirmozafari@iums.ac.ir

\section{Introduction}

Persisters are defined as a subpopulation of bacterial cells in a non-growing state characterized by a transient phenotype state (Abbasi et al. 2015). Persister cells can survive lethal doses of antibiotics, and this tolerance, contrary to resistance, is not associated with genetic inheritance (Narimisa et al. 2020). As evidenced, these subpopulations of bacteria show biphasic killing curves following exposure to high doses of antibiotics, while many other bacteria are rapidly killed. These population of bacteria are clinically important as they contribute to antibiotic tolerance and thereby leading to chronic infections and treatment failure (Fisher et al. 2017). Persister cell formation has been attributed to many mechanism, the most studied one being the toxin-antitoxin (TA) systems (Page et al. 2016). TA systems are defined as small genetic loci widely distributed among bacterial genomes. These systems are comprised of a stable toxin and its cognate labile antitoxin coded on a single operon. (Guglielmini et al. 2011). To date, six classes of these TA systems have been identified based on the mechanism of activity and the nature of TA components, with type II being the most studied type. Type II TA systems are comprised of protein toxins and antitoxins and have been repeatedly reported as players of bacterial stress responses (Goeders et al. 2014). Upon stress, the antitoxin is degraded by cellular proteases such as Lon or Clp protease, and the liberated toxin component interferes with vital cellular processes such as translation, cell-wall synthesis and replication, which subsequently affect bacterial growth and lead to the emergence of persisters (Coussens et al. 2016).

Persister cell formation has been demonstrated in almost all bacterial pathogens, one of them being Staphylococcus aureus (Chang et al. 2020). S. aureus is considered as one of the major antibiotic-resistant pathogens and a public health concern (Rajabi et al. 2020). Resistance to penicillin in this bacterium was reported early after the discovery of penicillin. Even after the development of other antibiotics such as methicillin, emergence and worldwide outbreaks of methicillin-resistant $S$. aureus was reported, becoming a threat to global health (Mwangi et al. 2007). Reportedly, these isolates are also resistant to other classes of antibiotics, such as vancomycin. Apart from resistance, persisters can lead to chronic infections 
Hosseini et al.

Table 1. Predicted toxin-antitoxin systems in this study.

\begin{tabular}{lllll}
\hline TA Type & Toxin & Antitoxin & Classification (family/domain) & Location on S. aureus N315 \\
\hline 2 & SA1873 & SAS067 & MazEF/RHH-MazF & $2121172 . .2121701$ \\
2 & SA2195 & SA2196 & relBE/PHD-RelE & $2466001 . .2466518$ \\
\hline
\end{tabular}

and treatment failure, thereby doubling the burden of these bacterial species (Singh et al. 2017). Therefore, it is crucial to study these subpopulations of bacteria and the related mechanisms. For this purpose, this study aimed the evaluation of persister cell formation in MRSA isolates following exposure to different antibiotics and investigation of the expression levels of type II TA systems in these bacteria.

\section{Materials and methods}

\section{Bacterial strains and growth conditions}

In this study, S. aureus subsp. aureus N315 and 5 clinical MRSA isolates were used. Isolates were previously collected from burn wounds and taxonomical classification was carried out by using biochemical and molecular tests. Bacterial strains were stored in Brain Heart Infusion (BHI) broth (Merck, Darmstadt, Germany) containing $20 \%$ glycerol at $-80{ }^{\circ} \mathrm{C}$.

\section{Determination of the Minimum Inhibitory Concentra- tion (MIC)}

MIC of ciprofloxacin (Sigma Aldrich, Germany) against S. aureus subsp. aureus N315 and MRSA isolates was determined by the broth microdilution assay using $96 \mathrm{U}$-shaped well plates (Singh et al. 2017). For this purpose, $100 \mu \mathrm{L}$ of Mueller-Hinton broth (Oxoid, UK) was added to each well of the plate, and $100 \mu \mathrm{L}$ of ciprofloxacin solution was added to the first well and serially diluted. Finally, 100 $\mu \mathrm{L}$ of the overnight culture containing $\sim 3 \times 10^{8}$ bacterial cells were added to each well. For MIC determination, the plate was incubated at $37^{\circ} \mathrm{C}$ for $24 \mathrm{~h}$ and bacterial growth was recorded. MIC values for ciprofloxacin was measured independently in triplicates.

\section{Persister formation}

To induce persister cells in the studied isolates, bacterial cultures in the exponential phase were exposed to high doses of ciprofloxacin. For quantification of the persister cells, a single colony of MRSA isolates or $S$. aureus subsp. aureus $\mathrm{N} 315$ was inoculated into $5 \mathrm{ml}$ BHI broth (Merck, Darmstadt, Germany) for $24 \mathrm{~h}$. Then, bacterial subculture was diluted $1: 100$ and incubated at $37^{\circ} \mathrm{C}$ on a shaker at $200 \mathrm{rpm}$ to obtain an optical density of 0.4 at $600 \mathrm{~nm}$. To draw bacterial killing curves, bacterial cultures in the exponential phase were exposed to 10-fold MIC concentration of ciprofloxacin, and cultures were grown at 37 ${ }^{\circ} \mathrm{C}(220 \mathrm{rpm})$ for $3.5 \mathrm{~h}$. After centrifugation at $1400 \mathrm{rpm}$ for 5 min, bacterial cultures were washed with PBS twice and after supernatant removal, fresh BHI broth was added and centrifuged at $220 \mathrm{rpm}$ at $37^{\circ} \mathrm{C}$. After ciprofloxacin stress, bacterial cells were washed twice by $0.85 \%$ sterile PBS for ciprofloxacin removal and after serial dilutions of bacterial culture, bacterial cells were inoculated onto BHI agar for CFU determination. Bacterial culture without ciprofloxacin exposure was selected as the control.

\section{Identification of TA system genes and their detection by polymerase chain reaction (PCR)}

Prediction of TA systems in S. aureus subsp. aureus N315 was carried out by using RASTA-bacteria and TADB2.0

Table 2. Primers used in this study.

\begin{tabular}{|c|c|c|c|}
\hline TA System & Sequence (5' - 3') & Annealing temperature $\left({ }^{\circ} \mathrm{C}\right)$ & Amplicon size (bp) \\
\hline \multirow[t]{2}{*}{ gyrB } & AGGTCTTGGAGAAATGAATG & 62 & 113 \\
\hline & CAAATGTTTGGTCCGCTT & & \\
\hline \multirow[t]{2}{*}{ SA1873 (T) } & AAGGGGGAGTCAGACCTGTA & 58 & 91 \\
\hline & ATCCTACCAGTTATTGCCGC & & \\
\hline \multirow[t]{2}{*}{ SAS067 (AT) } & TCAAAATAGAAGTCATAGCTTAGAACA & 57 & 157 \\
\hline & TCATTCATTCGTTGAATTAGAAGAT & & \\
\hline \multirow[t]{2}{*}{ SA2195 (T) } & ATTACGTTTTCGCCTCAAGC & 59 & 154 \\
\hline & TCAGATTCGATTTTAACTTTTCAGG & & \\
\hline \multirow[t]{2}{*}{ SA2196 (AT) } & CGCTCGACAGAATTTAAAGG & 58 & 152 \\
\hline & TTGGGTTCTGTTGGAGGTAAA & & \\
\hline
\end{tabular}




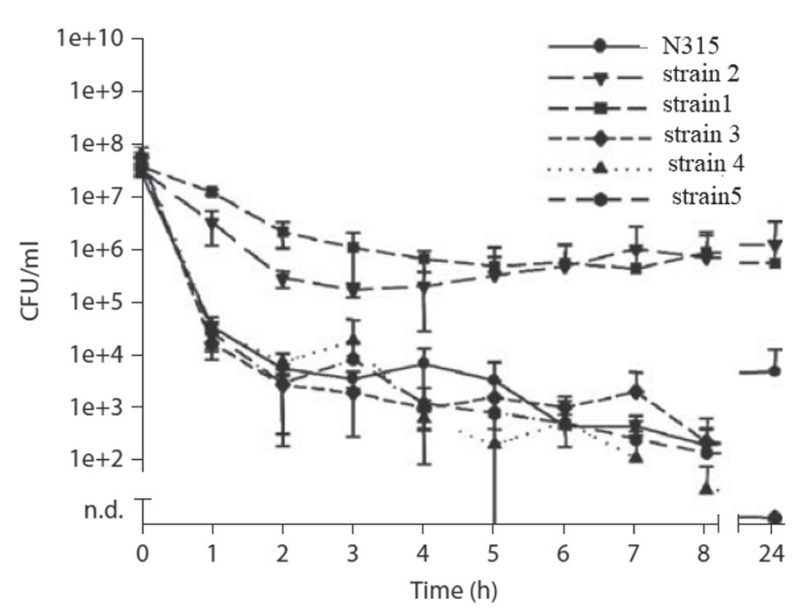

Figure 1. Time-dependent killing curve of the studied strains at exponential growth following exposure to $5 \mu \mathrm{g} / \mathrm{ml}$ of ciprofloxacin.

database (Sevin et al. 2007, Xie et al. 2018). Two TA loci including mazEF and relBE were predicted (Table 1). To investigate the presence of these TA systems in our studied isolates, we performed PCR assay using specific primers targeting these loci designed by Oligo 7 and Primer 3 (Table 2). For this purpose, total DNA of bacterial isolates was extracted using boiling method as previously described (Junior et al. 2016). A total final volume of $25 \mu \mathrm{L}$ of PCR reaction consisted of $12.5 \mu \mathrm{L}$ MasterMix (Amplicon, USA), $2 \mu \mathrm{L}$ of each primer (forward and reverse with the concentration of $10 \mathrm{pmol}), 2 \mu \mathrm{L}$ of template bacterial DNA, and $6.5 \mu \mathrm{L}$ sterile distilled water. Thermal cycling program consisted of initial denaturation for $5 \mathrm{~min}$ at 94 ${ }^{\circ} \mathrm{C}$, followed by 30 cycles of denaturation for $1 \mathrm{~min}$ at 94 ${ }^{\circ} \mathrm{C}$, annealing (based on Table 2) for $30 \mathrm{~s}$, extension for $1 \mathrm{~min}$ at $72^{\circ} \mathrm{C}$, and final extension for $5 \mathrm{~min}$ at $72{ }^{\circ} \mathrm{C}$. PCR amplicons were then analyzed by electrophoresis on $1.5 \%$ agarose gel.

\section{Relative RT-qPCR}

Total RNA was extracted from $1 \mathrm{ml}$ of the studied bacterial cultures in the exponential phase after $5 \mathrm{~h}$ of treatment with 100-fold MICs concentrations of ciprofloxacin. Total RNA of S. aureus that was not exposed to ciprofloxacin was also extracted as the control, using high pure RNA isolation kit (Roche, Germany) according to the protocols provided by the manufacturer. For removing DNA contamination, DNaseI treatment was carried out (Thermo Scientific, USA) according to the protocol. To assess RNA quality, NanoDrop spectrophotometer (Thermo Scientific, USA) and gel electrophoresis analysis were used. For cDNA synthesis, cDNA Synthesis Kit (Thermo Scientific, USA) was used. Primers used for real-time PCR was the same used for our PCR assay (Table 2). Quantitative reverse transcription-PCR (RT-qPCR) was carried out in triplicates for each sample using a Rotor-Gene thermal cycler (Corbett Life Sciences, Sydney, Australia) with SYBR Green method (Ampliqon Co, Denmark). Total 20 $\mu \mathrm{L}$ amplification reaction contained $2 \mu \mathrm{L}$ of cDNA, $10 \mu \mathrm{L}$ SYBR Green master mix, $7 \mu \mathrm{L}$ nuclease-free water, and $0.8 \mu \mathrm{L}$ of each forward and reverse primer. PCR reaction was as follows: an initial denaturation at $95^{\circ} \mathrm{C}$ for $1 \mathrm{~min}$, followed by 40 cycles at $95^{\circ} \mathrm{C}$ for $20 \mathrm{~s}$, annealing temperature (Table 2) for $25 \mathrm{~s}$, and $72{ }^{\circ} \mathrm{C}$ for $20 \mathrm{~s}$. gyrB was used as an internal control for normalization of the expression levels. The delta-delta Ct method was used for quantification of the relative fold changes in expression levels (Livak et al. 2001).

\section{Statistical analysis}

To assess significant differences in gene expression levels, T-Test and Mann-Whitney test were used. All data analyses and drawings were carried out with GraphPad Prism 8 (GraphPad Software).

\section{Results}

\section{Persister cell formation assays}

The MIC of ciprofloxacin was $0.5 \mu \mathrm{g} / \mathrm{ml}$. To study the ability of persister cell formation, we treated the studied bacterial cultures in the exponential phase with 10 -fold MIC Concentration of ciprofloxacin $(5 \mu \mathrm{g} / \mathrm{ml})$ and observed a rapid reduction in bacterial CFU values. This reduction rate varied from $94 \%$ for strain 1 to $99 \%$ for S. aureus subsp. aureus N315 after 5 h. Relatively constant CFU rate after $5 \mathrm{~h}$ up to $24 \mathrm{~h}$ was indicative of the presence of persister cells in response to ciprofloxacin exposure (Fig. 1).

\section{Presence of TA system genes and their relative expres- sion following antibiotic exposure}

According to the results of PCR assay, all the studied strains harbored both mazEF and relBE TA loci. The expression levels of $m a z F$ and relE genes increased in isolates exposed to ciprofloxacin ( $\mathrm{p}$-value $<0.05$ ). Also, $m a z F$ expression showed a higher increase compared to relE expression (Fig. 2).

\section{Discussion}

S. aureus isolates, and especially methicillin resistant S. aureus (MRSA), are considered as major pathogens that are responsible for nosocomial and communityacquired infections with high mortality rate, especially 


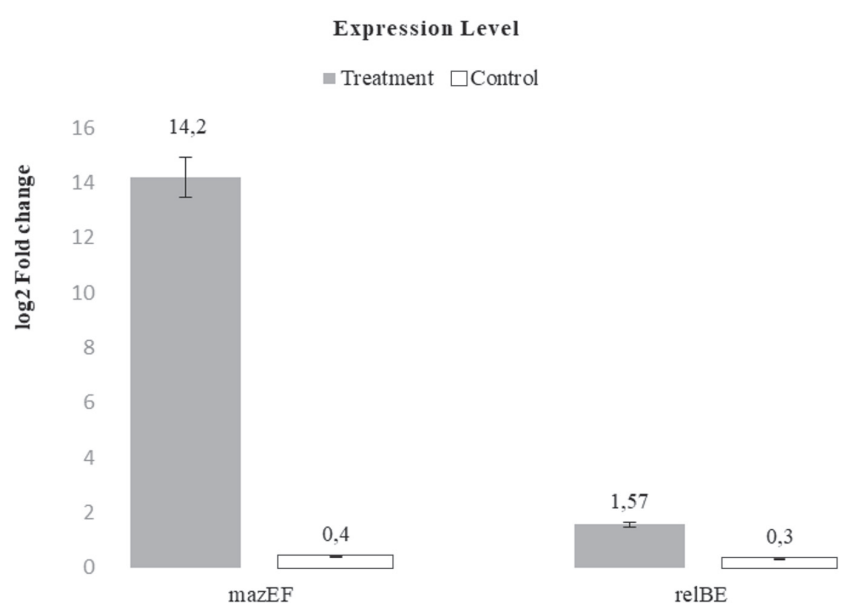

Figure 2. Expression levels of $m a z F$ and relE genes in the treatment and control groups.

in wound burn patients (Lee et al. 2018; Lakhundi et al. 2018). The major concern about infections caused by $S$. aureus is treatment failure of MRSA infections, leading to increased mortality rate and treatment costs (Turner et al. 2019; Gajdács 2019). Several studies have indicated the involvement of TA systems in bacterial persister cell formation following exposure to antibiotic stresses. Therefore, these systems could be potentially important for combating antibiotic tolerance in pathogenic bacteria (Wood 2016; Holden et al. 2018).

The effect of TA systems on persister cell formation was initially identified by Harris Moyed in 1983 . He indicated that two mutations in hipA could increase the rate of persister cell formation for 10000 fold (Moyed et al. 1983). Following this notion, the effect of TA system toxins in persister cell formation was investigated by gene deletion or overexpression. Vázquez et al. indicated that the overexpression of $r e l E$ and $m a z F$ could increase antibiotic tolerance and persister cell formation in E. coli strains (Vázquez-Laslop et al. 2006). Interestingly, a synergism has been indicated between protein toxins of TA systems and antibiotics. For instance, toxin component of the $\mathrm{CcdA} / \mathrm{CcdB}$ and ParE/ParD systems are involved in inhibiting DNA amplification by affecting DNA gyrase, which is also a target for quinolones. Also, RelE/ RelB and Phd/Doc systems affect ribosomal tRNA ${ }^{\text {fmet }}$, thereby disrupting protein synthesis. Similarly, antibiotics including macrolides and clindamycin target the 50S subunit of bacterial ribosome, thereby inhibiting protein biosynthesis. Considering the overlap between TA system toxin and antibiotics as well as their role in programmed cell death, novel antimicrobial agents can be developed with the ability to disrupt the balance between toxins and their cognate antitoxins. Also, considering the role of TA systems in antibiotic tolerance, biofilm formation, and pathogenicity, these systems can be suitable targets to combat pathogenic bacteria. As an illustration, it has been previously shown that mutation in hipBA TA system of $E$. coli modulates persister cell formation (Butt et al. 2014).

According to RT-qPCR results, $m a z F$ and relE expression increased for 35.3- and 5.24-fold, respectively, compared to the control group, indicating their possible role in persister cell formation ( $\mathrm{p}$-value $<0.05$ ). Several previous studies have indicated the involvement of MazF toxin in bacterial stress responses. MazEF system is generally one of the most studied TA systems in bacteria. For instance, in a study on E. coli, Engelberg-Kulka et al (2005) showed that this system is in fact a chromosomally encoded conserved loci with mRNA endonuclease activity that initiates programmed cell death in response to stress conditions, and finally retains a subpopulation of bacteria that can survive the harsh conditions (Engelberg-Kulka et al. 2005). Also, in another study on Listeria monocytogenes in 2018, Kalani et al. indicated that MazF toxin is activated in heat stress and therefore, it can contribute to bacterial survival (Kalani et al. 2018). In another study on L. monocytogenes, it was demonstrated that MazEF was activated during antibiotic stress, and is essential for bacterial survival (Curtis et al. 2017).

RelBE is another TA system in E. coli that has been extensively studied. This system is involved in bacterial response to amino acid deprivation and leads to the inhibition of translation. In a study conducted by Coskun et al. (2018), it was reported that TA system genes were encoded in the genome of Pseudomonas aeruginosa and $S$. aureus and all MRSA isolates $(\mathrm{n}=78)$ harbored relBE and higBA TA loci (Coskun 2018), which was in agreement to our study.

In a study by Butt and co-workers, it was shown that the exposure of Burkholderia pseudomallei to ciprofloxacin could increase HicA toxin levels and subsequently led to persister cell formation (Butt et al. 2014). Other studies have indicated that bactericidal antibiotics such as kanamycin have the ability to inhibit TA systems in E. coli. These TA systems included MazE/MazF, RelB/ RelE, MqsR/MqsA, and BigB/BigA (Hu et al. 2010). In another study on the expression of TA system genes in E. coli, following exposure to kanamycin and ampicillin, all the mentioned TAs systems were inhibited, indicating that cell death following treatment with bactericidal antibiotics could be associated with many TA systems (Lee and Lee 2016).

\section{References}

Abbasi K, Tajbakhsh E (2015) Prevalence of Shiga toxin 
genes and intimin genes in uropathogenic Escherichia coli. J Coast Life Med 3:791-796.

Butt A, Higman V, Williams C, Crump M, Hemsley C, Harmer N, Titball R (2014) The HicA toxin from Burkholderia pseudomallei has a role in persister cell formation. Biochem J 459:333-344.

Chang J, Lee R, Lee W (2020) A pursuit of Staphylococcus aureus continues: a role of persister cells. Arch Pharm Res 1-9.

Coskun U (2018) Effect of mazEF, higBA and relBE toxinantitoxin systems on antibiotic resistance in Pseudomonas aeruginosa and Staphylococcus isolates. Malawi Med J 30:67-72.

Coussens N, Daines D, (2016) Wake me when it's over-bacterial toxin-antitoxin proteins and induced dormancy. Exp Biol Med 241:1332-1342.

Curtis T, Takeuchi I, Gram L, Knudsen G (2017) The influence of the toxin/antitoxin mazEF on growth and survival of Listeria monocytogenes under stress. Toxins 9:31.

Engelberg-Kulka H, Hazan R, Amitai S (2005) mazEF: a chromosomal toxin-antitoxin module that triggers programmed cell death in bacteria.J Cell Sci 118:4327-4332.

Fisher R, Gollan B, Helaine S (2017) Persistent bacterial infections and persister cells. Nat Rev Microbiol 15(8):453-464.

Gajdács M (2019) The continuing threat of methicillinresistant Staphylococcus aureus. Antibiotics 8(2):52.

Goeders N, Van Melderen L (2014) Toxin-antitoxin systems as multilevel interaction systems. Toxins 6:304-324.

Guglielmini J, Van Melderen L (2011) Bacterial toxin-antitoxin systems: Translation inhibitors everywhere. Mob Genet Elements 1:283-306.

Holden D, Errington J (2018) Type II toxin-antitoxin systems and persister cells. MBio 9(5):e01574-18.

Hu MX, Zhang X, Li EL, Feng Y-J (2010) Recent advancements in toxin and antitoxin systems involved in bacterial programmed cell death. Int J Microbiol 2010:781430.

Junior J, Tamanini R, Soares B, de Oliveira A, de Godoi Silva F, da Silva F, Augusto N, Beloti V (2016) Efficiency of boiling and four other methods for genomic DNA extraction of deteriorating spore-forming bacteria from milk. Semin Cienc Agrar 37:3069-3078.

Kalani B, Irajian G, Lotfollahi L, Abdollahzadeh E, Razavi S (2018) Putative type II toxin-antitoxin systems in Listeria monocytogenes isolated from clinical, food, and animal samples in Iran. Microb Pathog 122:19-24.

Lakhundi S, Zhang K (2018) Methicillin-resistant Staphylococcus aureus: molecular characterization, evolution, and epidemiology. Clin Microbiol Rev 31(4):e00020-18.

Lee AS, de Lencastre H, Garau J, Kluytmans J, MalhotraKumar S, Peschel A, Harbarth S (2018) Methicillin-re- sistant Staphylococcus aureus. Nat Rev Dis Primers 4:1-23.

Lee K, Lee B (2016) Structure, biology, and therapeutic application of toxin-antitoxin systems in pathogenic bacteria. Toxins 8:305.

Livak K, Schmittgen T (2001) Analysis of relative gene expression data using real-time quantitative PCR and the $2-\Delta \Delta \mathrm{CT}$ method. Methods 25:402-408.

Moyed H, S. Bertrand K (1983) hipA, a newly recognized gene of Escherichia coli K-12 that affects frequency of persistence after inhibition of murein synthesis. J Bacteriol 155:768-775.

Mwangi M, Wu, Zhou Y, Sieradzki K, de Lencastre H, Richardson P, Bruce D, Rubin E, Myers E, Siggia E (2007) Tracking the in vivo evolution of multidrug resistance in Staphylococcus aureus by whole-genome sequencing. PNAS 104:9451-9456.

Narimisa N, Kalani B. S, Amraei F, Mohammadzadeh R, Mirkalantari S, Razavi S, Jazi F (2020) Type II toxin/ antitoxin system genes expression in persister cells of Klebsiella pneumoniae. Rev Med Microbiol 31(4):215-220.

Page R, Peti W (2016) Toxin-antitoxin systems in bacterial growth arrest and persistence. Nat Chem Biol 12:208214.

Rajabi S, Shivaee A, Khosravi A, Eshaghi M, Shahbazi S, Hosseini F (2020) Evaluation of multidrug efflux pump expression in clinical isolates of Staphylococcus aureus. Gene Rep 18:100537.

Sevin E, Barloy-Hubler F (2007) RASTA-Bacteria: a webbased tool for identifying toxin-antitoxin loci in prokaryotes. Genome Biol 8:R155.

Singh M, Matsuo M, Sasaki T, Morimoto Y, Hishinuma T, Hiramatsu K (2017) In vitro tolerance of drug-naive Staphylococcus aureus strain FDA209P to vancomycin. Antimicrob Agents Chemother 61(2):e01154-16.

Turner N, Sharma-Kuinkel B, Maskarinec S, Eichenberger E, Shah P, Carugati M, Holland T, Fowler G (2019) Methicillin-resistant Staphylococcus aureus: an overview of basic and clinical research. Nat Rev Microbiol 17:203-218.

Vázquez-Laslop N, Lee H, Neyfakh A (2006) Increased persistence in Escherichia coli caused by controlled expression of toxins or other unrelated proteins. J Bacteriol 188:3494-3497.

Wood T (2016) Combatting bacterial persister cells. Biotechnol Bioeng 113:476-483.

Xie Y, Wei Y, Shen Y, Li X, Zhou H, Tai C, Deng Z, Ou H (2018) TADB 2.0: an updated database of bacterial type II toxin-antitoxin loci. Nucleic Acids Res 46:D749-D753. 
